\title{
Aseptic Meningitis With Systemic Lupus Erythematosus: Case Report and Review of the Literature
}

\author{
Masako TSUKAMOTO@, Masaki SHIMAMOTO®, Takeshi TERASHIMA®, Noriyuki SETA® \\ Department of Internal Medicine, Tokyo Dental College Ichikawa General Hospital, Ichikawa, Japan
}

\begin{abstract}
In this article, we describe a 29-year-old female patient with a history of systemic lupus erythematosus (SLE) in remission for six years presenting with fever, headache, and arthritis. Neurological examination was normal, as were brain computed tomography and magnetic resonance imaging, but based on results of cerebrospinal fluid examination and symptoms indicating active SLE, including erythema and oligoarticular arthritis, she was diagnosed as aseptic meningitis secondary to SLE recurrence. Her symptoms promptly improved with prednisolone treatment. This case suggests that aseptic meningitis should be considered in SLE patients, even when neurological findings are equivocal.

Keywords: Aseptic; central nervous system; meningitis; neuropsychiatric; systemic lupus erythematosus.
\end{abstract}

Systemic lupus erythematosus (SLE) is a complex autoimmune systemic disease. Patients with SLE may experience a variety of neurological and psychiatric manifestations, collectively named neuropsychiatric SLE (NPSLE), that account for significant morbidity and mortality. Neuropsychiatric symptoms are encountered in $21-95 \%$ of patients with SLE and can be directly related to central nervous system (CNS) manifestations. ${ }^{1-3}$ Meningitis is a rare CNS manifestation, observed in 1.4-2.0\% of patients with SLE. ${ }^{4-6}$ It can be either septic or aseptic, almost $50 \%$ being aseptic. ${ }^{5,7}$ Aseptic meningitis in patients with SLE is mainly caused by nonsteroidal anti-inflammatory drugs, such as ibuprofen and immunosuppressants. ${ }^{8-9}$ Cerebrospinal fluid (CSF) examination is necessary for the diagnosis of meningitis, and definitely indicated in the presence of signs of meningeal irritation, such as neck stiffness,
Kernig's and Brudzinski's signs. In this article, we report a case of aseptic meningitis as a manifestation of recurrence of SLE in a patient not receiving nonsteroidal anti-inflammatory drugs or immunosuppressants and with negative neurologic examination.

\section{CASE REPORT}

A 29-year-old female patient with a history of SLE was admitted to our hospital because of fever, bilateral knee pain, and headache. Six years previously, she was diagnosed with SLE manifested by facial erythema, oral ulcers, photosensitivity, arthritis, headache, leukocytopenia, thrombocytopenia, and antinuclear antibody and anti-Smith antibody positivity. Anti-double stranded deoxyribonucleic acid antibody, anti-U1-ribonucleoprotein antibody

Received: May 24, 2018 Accepted: July 11, 2018 Published online: September 04, 2018

Correspondence: Noriyuki Seta, MD. Department of Internal Medicine, Tokyo Dental College Ichikawa General Hospital, 272-8513 Ichikawa, Japan. Tel: 81-47-322-0151 e-mail: nseta@tdc.ac.jp 
or other autoantibodies was undetected. She did not have any neurological symptoms. She was subsequently treated with $30 \mathrm{mg} /$ day of prednisolone and achieved remission. After gradual tapering of prednisolone, clinical and serological remission was maintained by a maintenance dose (5 mg/day) of prednisolone. Six years after the diagnosis of SLE, she developed fever, joint pain, and headache. An outpatient clinic blood test indicated a high level of C-reactive protein (CRP) and she was therefore admitted to our hospital. Physical examination revealed normal blood pressure $(124 / 80 \mathrm{mmHg})$. She was febrile $\left(39.5^{\circ} \mathrm{C}\right)$. She received paracetamol before admitting to our hospital, but her fever did not improve. Findings of ocular, lung, cardiovascular, abdominal, and neurological examinations were normal, but swelling and tenderness of both knee joints and facial erythema were noted. Neck stiffness, Kernig's and Brudzinski's signs and other neurological findings suggestive of meningeal irritation were absent. Laboratory tests revealed moderate thrombocytopenia (blood platelet count $86,000 / \mu \mathrm{L}$ ) and a high level of CRP $(8.76 \mathrm{mg} / \mathrm{dL})$. There was no increase in circulating immune complexes as measured by serum Clq binding activity, and serum complement levels were normal. Other blood parameters, including levels of liver enzymes, blood glucose, white blood count and hemoglobin, were within normal ranges. CSF examination revealed 107 leukocytes $/ \mathrm{mm}^{3}$ with $47 \%$ mononuclear cells, glucose of $54 \mathrm{mg} / \mathrm{dL}$, and protein of $75.2 \mathrm{mg} / \mathrm{dL}$. CSF smears, cultures for bacteria and fungi, and virus antigen determinations for influenza virus, Herpes simplex virus, Varicella zoster virus, cytomegalovirus, Coxsackie virus, and EpsteinBarr virus were negative. Computed tomography and magnetic resonance imaging (MRI) scans of the brain were interpreted as normal. A written informed consent was obtained from the patient.

She was diagnosed with meningitis and received antibiotic (clarithromycin and ceftriaxone) for five days, but her symptoms did not improve. Then, we finally diagnosed aseptic meningitis as recurrence of SLE on the basis of the results of CSF examination and the presence of erythema and arthritis indicating active SLE. The antibiotic treatment was discontinued, and treatment with $30 \mathrm{mg} /$ day of prednisolone was initiated, followed by prompt defervescence and relief of the headache.
The bilateral knee arthritis also disappeared in several days and the level of CRP decreased to $0.03 \mathrm{mg} / \mathrm{dL}$ after 15 days. She was treated with hydroxychloroquine and clinical and serological remission was maintained during the observation.

\section{DISCUSSION}

In this article, we describe exacerbation of SLE presenting as a case of aseptic meningitis with oligoarticular arthritis, headache, and facial erythema. Aseptic meningitis is a rare neurologic manifestation of SLE. ${ }^{4-6}$ It can be induced by the disease itself, as a reaction to medications or infection such as virus and is diagnosed by CSF examination and clinical information. Our case had fever and headache but no meningeal irritation signs such as neck stiffness, Kernig's or Brudzinski's signs, and no other neurological findings. We diagnosed her as aseptic meningitis due to recurrence of SLE on the basis of the SLE symptoms of erythema and oligoarticular arthritis, the results of CSF examination, and the response to steroid therapy.

It is difficult to diagnose meningitis with SLE and distinguish other NPSLE including lupus headache or vasculitis. We established a diagnosis of aseptic meningitis with fever, headache, and CSF findings. It is known that patients with aseptic meningitis show significantly lower leukocytes and proteins in CSF compared to those with septic meningitis. ${ }^{5}$ Moreover, it has been reported that clinical findings, particularly psychiatric manifestations and abnormalities on brain MRI, are less common in SLE patients with aseptic meningitis. ${ }^{7}$ Therefore, compared to septic meningitis, a high level of clinical suspicion of aseptic meningitis can be necessary for diagnosis.

Some case series have been reported of aseptic meningitis caused by SLE itself (Table 1).10-14 Aseptic meningitis may develop either at onset or several years or months after SLE onset. Most patients presented with fever and headache, while two cases (cases 8 and 9, Table 1 ) $^{14}$ only had neurological signs. All patients were diagnosed with meningitis by CSF examination and received steroid therapy. Two cases (cases 4 and 5, Table 1$)^{11}$ had not been diagnosed as SLE at the onset of meningitis and their meningitis was promptly improved before treatment. However, 


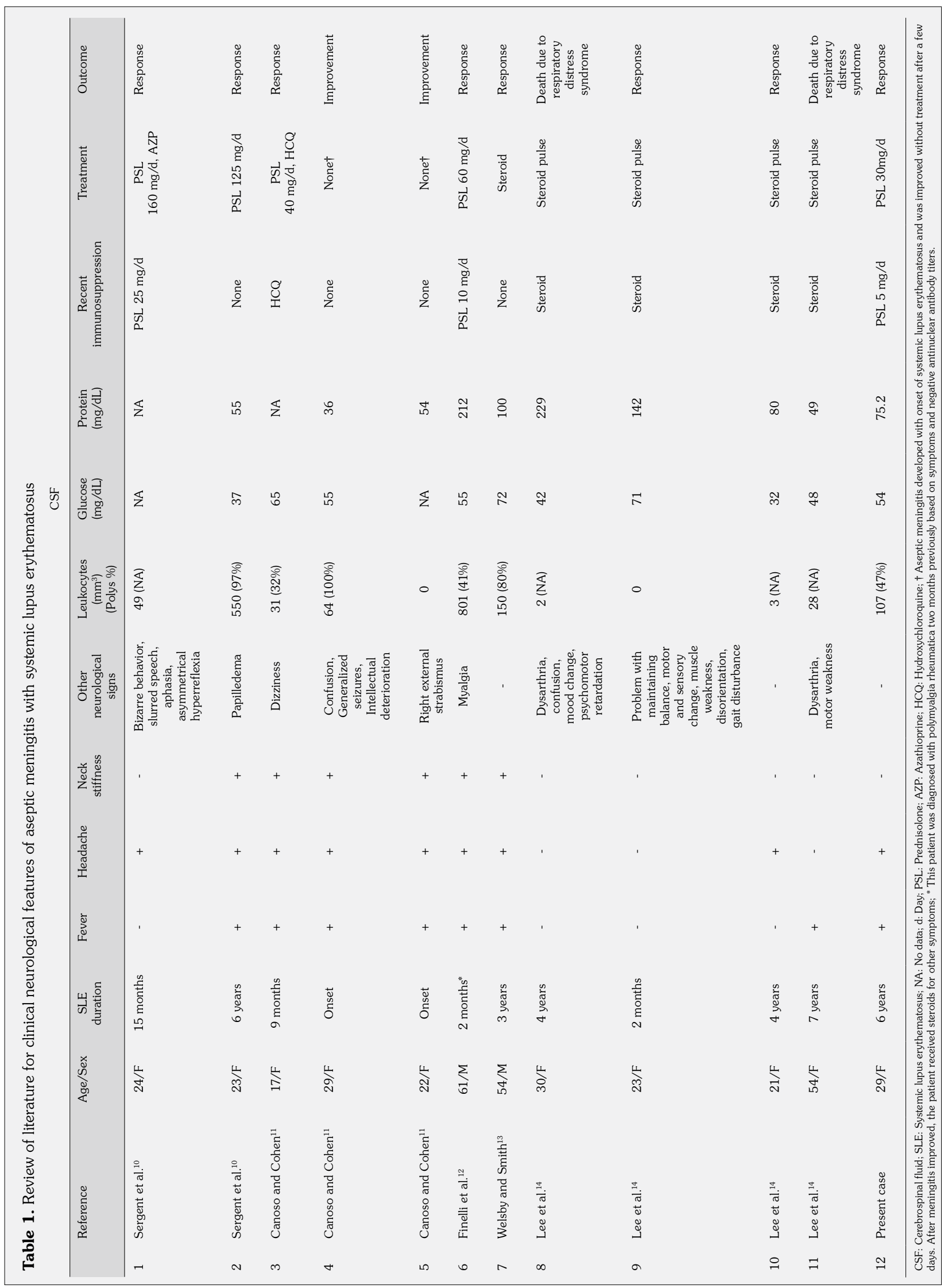


they received steroid treatment after a couple of days because they were diagnosed as SLE due to other symptoms after improving meningitis. The symptoms of 10 patients, including our case, promptly responded to therapy, but two case (Case 8 and 11, Table 1$)^{14}$ died from respiratory distress syndrome. In a previous review, of all patients (10/10) presenting with neck stiffness, psychiatric symptoms were shown in four patients (4/10) with aseptic meningitis caused by SLE itself. ${ }^{5}$ However, another review showed that only half of the patients with aseptic meningitis had neck stiffness, and none had psychiatric symptoms. ${ }^{7}$ In our case, the patient had fever and headache with no neck stiffness or neurological or psychiatric symptoms. Therefore, neurological and psychiatric symptoms may be absent in aseptic meningitis caused by SLE itself, necessitating a high level of clinical suspicion for diagnosis.

\section{Declaration of conflicting interests}

The authors declared no conflicts of interest with respect to the authorship and/or publication of this article.

\section{Funding}

The authors received no financial support for the research and/or authorship of this article.

\section{REFERENCES}

1. Zirkzee EJ, Huizinga TW, Bollen EL, van Buchem MA, Middelkoop HA, van der Wee NJ, et al. Mortality in neuropsychiatric systemic lupus erythematosus (NPSLE). Lupus 2014;23:31-8.

2. Brey RL, Holliday SL, Saklad AR, Navarrete MG, Hermosillo-Romo D, Stallworth CL, et al. Neuropsychiatric syndromes in lupus: prevalence using standardized definitions. Neurology 2002;58:1214-20.

3. Hanly JG, McCurdy G, Fougere L, Douglas JA, Thompson K. Neuropsychiatric events in systemic lupus erythematosus: attribution and clinical significance. J Rheumatol 2004;31:2156-62.

4. Kasitanon N, Louthrenoo W, Piyasirisilp S, Sukitawu W, Wichainun R. Neuropsychiatric manifestations in Thai patients with systemic lupus erythematosus. Asian Pac J Allergy Immunol 2002;20:179-85.

5. Baizabal-Carvallo JF, Delgadillo-Márquez G, Estañol B, García-Ramos G. Clinical characteristics and outcomes of the meningitides in systemic lupus erythematosus. Eur Neurol 2009;61:143-8.

6. Feinglass EJ, Arnett FC, Dorsch CA, Zizic TM, Stevens MB. Neuropsychiatric manifestations of systemic lupus erythematosus: diagnosis, clinical spectrum, and relationship to other features of the disease. Medicine (Baltimore) 1976;55:323-39.

7. Kim JM, Kim KJ, Yoon HS, Kwok SK, Ju JH, Park $\mathrm{KS}$, et al. Meningitis in Korean patients with systemic lupus erythematosus: analysis of demographics, clinical features and outcomes; experience from affiliated hospitals of the Catholic University of Korea. Lupus 2011;20:531-6.

8. Widener HL, Littman BH. Ibuprofen-induced meningitis in systemic lupus erythematosus. JAMA 1978;239:1062-4.

9. Sergent JS, Lockshin M. Azathioprine-induced meningitis in systemic lupus erythematosus. JAMA 1978;240:529.

10. Sergent JS, Lockshin MD, Klempner MS, Lipsky BA. Central nervous system disease in systemic lupus erythematosus. Therapy and prognosis. Am J Med 1975;58:644-54.

11. Canoso JJ, Cohen AS. Aseptic meningitis in systemic lupus erythematosus. Report of three cases. Arthritis Rheum 1975;18:369-74.

12. Finelli PF, Yockey CC, Herbert AJ Jr. Recurrent aseptic meningitis in an elderly man. Unusual prodrome of systemic lupus erythematosus. JAMA 1976;235:1142-3.

13. Welsby P, Smith C. Recurrent sterile meningitis as a manifestation of systemic lupus erythematosis. Scand $\mathrm{J}$ Infect Dis 1977;9:149-50.

14. Lee JH, Lee JY, Lee YJ, Park DW, Kim YS, Kim HY. Noninfectious Meningitis Caused by Systemic Lupus Erythematosus: A Case Series of 4 Patients. J Comput Assist Tomogr 2016;40:424-7. 